\title{
A Modification to the Behavioural Regulation in Exercise Questionnaire to Include an Assessment of Amotivation
}

\author{
David Markland and Vannessa Tobin \\ University of Wales
}

\begin{abstract}
Drawing on self-determination theory, Mullan, Markland, and Ingledew (1997) developed the Behavioural Regulation in Exercise Questionnaire (BREQ) to measure the continuum of behavioral regulation in exercise contexts. The BREQ assesses external, identified, introjected, and intrinsic regulations. Mullan et al. initially included a set of amotivation items but dropped these due to high levels of skewness and a restricted response range in their development sample. It would clearly be useful to assess amotivation for exercise. This study aimed to test the factorial validity of a modified BREQ with amotivation items reinstated in a sample likely to exhibit a wider range of amotivation responses. A total of 194 former exercise referral scheme participants completed the revised instrument (BREQ-2). Although the amotivation items were still skewed, confirmatory factor analysis using the Satorra-Bentler (1994) scaling correction to $\chi^{2}$ indicated an excellent model fit. The BREQ- 2 could prove useful to researchers wishing to assess amotivation in order to develop a more complete understanding of motivation for exercise.
\end{abstract}

Key Words: exercise motivation, measurement, self-determination theory

Self-determination theory (SDT: Deci \& Ryan, 1985, 1991) is a popular theoretical framework for the investigation of motivation in exercise psychology. One aspect of the theory that has generated particular interest is its multidimensional conceptualization of intrinsic and extrinsic motivation. According to SDT, there are varying forms of motivation that represent qualitatively different ways in which a behavior can be regulated. The theory proposes that these forms of regulation lie along a continuum ranging from completely non-self-determined to completely self-determined regulation. The six different forms of regulation are labeled: amotivation, external regulation, introjection, identification, integration, and intrinsic regulation.

Amotivation is a state of lacking any intention to engage in a behavior and is a completely non-self-determined form of regulation. External regulation involves engaging in a behavior only in order to satisfy external pressures or to achieve externally imposed rewards. Introjected regulation involves the internalization of external controls, which are then applied through self-imposed pressures in order to

The authors are with the School of Sport, Health and Exercise Sciences, University of Wales, Bangor, George Bldg, Holyhead Rd, Bangor, Gwynedd, Wales, UK, LL57 2PX. 


\section{2 / Markland and Tobin}

avoid guilt or to maintain self-esteem. Identification involves a conscious acceptance of the behavior as being important in order to achieve personally valued outcomes. Integrated regulation concerns the assimilation of identified regulation so that engaging in the behavior is fully congruent with one's sense of self. Intrinsic regulation involves taking part in an activity for the enjoyment and satisfaction inherent in engaging in the behavior itself.

Mullan, Markland, and Ingledew (1997) developed an instrument to measure the different forms of behavioral regulation in exercise contexts. The Behavioural Regulation in Exercise Questionnaire (BREQ) assesses external, identified, introjected, and intrinsic regulations. Confirmatory factor analysis supported the factorial validity of the instrument in a sample of leisure center users and its factorial validity and invariance across gender in a worksite sample (Mullan et al., 1997). Mullan and Markland (1997) found that the BREQ discriminated between individuals at different stages of change for exercise, with those in the later stages being more self-determined in their behavioral regulation than those in the earlier stages. Rose, Markland, and Parfitt (2001) reported evidence supporting the convergent and discriminant validity of the BREQ subscales with respect to exercise causality orientations, also in a worksite sample. Wilson, Rodgers, and Fraser (2002), also using confirmatory factor analysis, provided further support for the factorial validity of the BREQ and for its convergent validity with respect to conceptually related scales and exercise behavior in a sample of university students and staff. Wilson, Rodgers, Blanchard, and Gesell (2003) found further evidence for the convergent, discriminant, and predictive validity of the BREQ in relation to conceptually related scales, exercise behavior, and physical fitness in a longitudinal study.

There is growing evidence, then, for the validity of the BREQ as a measure of the continuum of behavioral regulation in exercise contexts. However, in the initial development of the measure, Mullan et al. (1997, Study 1) found that items designed to tap amotivation exhibited very high levels of skewness and a restricted range of scores, and so these items were eliminated from subsequent analyses. A likely explanation for this was that most of the participants in the initial validation study were attendees at a leisure center who were exercising regularly. One would not expect such individuals to be amotivated for exercise. Clearly it would be theoretically useful to include a valid measure of amotivation in the assessment of behavioral regulations for exercise in order to investigate the antecedents and consequences of amotivation. This study aimed to test the factorial validity of a revised BREQ (BREQ-2), which included amotivation items in a sample that was likely to present a wider range of responses to such items.

\section{Method}

\section{Sample and Procedure}

Respondents were individuals who had taken part in an exercise referral scheme during the previous 3 years. In such schemes in the U.K., health care professionals refer patients whom they consider would benefit from adopting a more active lifestyle, and who meet at least one of a set of participation criteria, usually including overweight/obesity, moderate hypertension, and mild depression. The emphasis is normally on referring individuals at low to moderate risk of morbidity/mortality rather than those in higher risk categories such as cardiac 
rehabilitation patients. Participants undergo a medical examination and are referred to a community leisure center where they receive a fitness assessment, an exercise prescription, and a series of exercise sessions. Typically, 10 to 12 sessions are offered at a reduced cost or for free.

Following ethics approval from a health trust, 580 questionnaires were sent to potential participants along with an explanatory letter, an informed consent form, and a prepaid reply envelope. This was close to the total number of referrals made within the 3 years of the scheme's operation. A total of 201 questionnaires (35\%) were returned: $68 \%$ from women (mean age $54.24 \mathrm{yrs}, S D=13.28$ ) and $30 \%$ from men (mean age 56.33 yrs, $S D=12.90$ ); the remaining $2 \%$ did not disclose their sex. Men and women did not differ significantly by age, $t(195)=1.03, p>0.05$. The sample was moderately physically active, as indicated by scores on Godin and Shephard's (1985) Leisure Time Exercise Questionnaire (mean METs $=31.14, S D=$ 24.03; see Instruments section). The sample was at the upper end of the overweight category of the body mass index (mean $=28.50 \mathrm{~kg} / \mathrm{m}^{2}, S D=5.94$ ), as would be expected in this population. In terms of occupation, $41 \%$ were retired, $15 \%$ were housewives, $9 \%$ care workers, $9 \%$ clerical, $7 \%$ manual, $6 \%$ unemployed/sick, and $13 \%$ other miscellaneous categories.

\section{Instruments}

The BREQ-2 comprised the four subscales of Mullan et al.'s (1997) BREQ assessing external (4 items, e.g., "I exercise because other people say I should"); introjected (3 items, e.g. "I feel guilty when I don't exercise"); identified (3 items, e.g., "I value the benefits of exercise"); and intrinsic (4 items, e.g., "I exercise because it's fun") regulations. In addition, 4 amotivation items from Mullan et al.'s (1997) initial item pool were included ("I don't see why I should have to exercise"; "I can't see why I should bother exercising"; "I don't see the point in exercising"; and "I think that exercising is a waste of time"). Due to an error, one item was omitted from the original BREQ identified subscale ("I get restless if I don't exercise regularly"). Responses were scored on a 5-point scale ranging from $0=$ "not true for me" to 4 = "very true for me."

Respondents also completed a modified version of Godin and Shephard's (1985) Leisure Time Exercise Questionnaire. This 3-item scale asks respondents to indicate the frequency of mild, moderate, and strenuous exercise undertaken in a typical week. These scores are weighted by metabolic equivalents $(3,5$, and 9 , respectively) and summed to yield an overall weekly activity score (METs). Respondents also provided demographic and personal information (age, sex, weight, height, occupation) and completed exploratory scales designed to tap perceptions of support in referral contexts; the data from these latter scales are not reported here.

\section{Analysis and Results}

The factorial validity of the BREQ-2 was assessed using confirmatory factor analysis (CFA) with LISREL 8.51. The covariance matrix was analyzed. The specified model allowed the items to load on their intended factors, interfactor correlations and error variances were also free to be estimated, and error covariances were constrained to zero. Listwise deletion of cases with missing data produced an effective sample size of 194. Preliminary analysis revealed that the data departed 


\section{4 / Markland and Tobin}

significantly from multivariate normality. Consequently, model fit was assessed using the Satorra-Bentler (1994) scaling correction to the maximum likelihood $\chi^{2}$. In addition, the root mean square error of approximation (RMSEA) and its $90 \%$ confidence interval, the comparative fit index (CFI), the non-normed fit index (NNFI), and the standarized root mean square residual (SRMR) were examined.

The results showed that the hypothesized 5-factor model did not depart significantly from the data (Satorra-Bentler $\chi^{2}=136.49$ [125], $p=.23$ ). The other fit indices also indicated an excellent fit (RMSEA $=.02,90 \% \mathrm{CI}=.00-.04 ; \mathrm{CFI}=$ $.95 ; \mathrm{NNFI}=.94 ;$ SRMR $=.05)$. Standardized factor loadings were all significant and moderate to strong $(M=.76$; range $.53-.90 ; p$ 's $<.001)$. Table 1 shows the means (calculated as the mean of the item scores for each subscale) and standard deviations of the subscales, factor intercorrelations, and Cronbach's alpha reliability coefficients. Internal consistency of all factors was acceptable.

Table 1 Mean Subscale Scores, Factor Intercorrelations, and Cronbach's Alpha Reliability Coefficients (on the diagonal) for BREQ-2 Subscales

\begin{tabular}{lcccrrrr}
\hline & $M$ & $S D$ & 1 & 2 & 3 & 4 & 5 \\
\hline Intrinsic & 2.80 & 1.02 & 0.86 & & & & \\
Identified & 3.24 & 0.87 & 0.80 & 0.73 & & & \\
Introjected & 1.74 & 1.25 & 0.08 & 0.25 & 0.80 & & \\
External & 0.59 & 0.90 & -0.59 & -0.48 & 0.08 & 0.79 & \\
Amotivation & 0.30 & 0.68 & -0.62 & -0.79 & -0.20 & 0.65 & 0.83 \\
\hline
\end{tabular}

\section{Discussion}

The results of this study showed that the addition of amotivation items to the original BREQ produced a model that had an excellent fit to the data, indicating that the new version has good factorial validity. However, a principal aim of the study was to assess the addition of a measure of amotivation in a sample that was likely to express a greater range of amotivation scores than that used in the preliminary development of the instrument. In fact, the mean for the amotivation subscale indicates low levels of amotivation and the distribution of scores on the amotivation items was heavily skewed - though not to the same extent as in Mullan et al.'s study (1997) — and the distribution did not exhibit the restricted range evidenced in their sample of regular exercisers.

A likely explanation for the skewed distribution here is that the respondents in this study had been through an exercise referral scheme. The fact that they had voluntarily participated in the scheme in itself suggests that the respondents would have had at least some motivation for exercise. Indeed, the present sample was moderately physically active according to the self-reported activity scale. Thus our initial expectation that such a sample would exhibit a wider range of amotivation scores was unwarranted. 
According to SDT, amotivation can arise from a lack of perceived contingency between behavior and desirable outcomes, a failure to value the behavior, or a feeling that one is not competent to successfully engage in it (Ryan \& Deci, 2000). The current BREQ-2 amotivation items assess mainly a failure to value exercise, although arguably they also implicitly tap a lack of perceived contingency between exercising and desired outcomes. For example, endorsing the item "I think exercising is a waste of time" carries the assumption that exercising is not rewarding as well as not valued. The instrument's authors did not develop items to assess a lack of perceived competence because they felt that the inclusion of such items might confound amotivation with perceived competence. Should distributional problems similar to those reported here be found in samples that do include more amotivated individuals, however, then further item development might be worthwhile in order to broaden the content of the amotivation subscale. We would like to acknowledge the contribution of an anonymous reviewer for this suggestion.

It should be noted that the mean for external regulation was also well below the scale's midpoint, suggesting that the sample had internalized the initial referral to exercise by external agencies to some extent. Furthermore, some caution is warranted in interpreting the results, due to the relatively small sample size. Nevertheless, the results of the CFA reported here suggest that the BREQ-2 has strong factorial validity and could prove useful for researchers wishing to assess amotivation along with the other forms of behavioral regulation for exercise.

As predicted by SDT (Ryan \& Connell, 1989), the correlations among the subscales generally conformed to a simplex-like pattern, with stronger positive correlations between factors adjacent on the self-determination continuum and stronger negative correlations between more distal factors. However, this was not the case for the correlations between amotivation and intrinsic and identified regulation. There was a stronger negative relationship between amotivation and identification than between amotivation and intrinsic regulation. This is perhaps not surprising because the identification items concern the personal importance or value placed on exercising whereas, as noted above, the amotivation items reflect a lack of such importance or value.

A potential concern here is that the identification and amotivation subscales could be assessing a single construct. However, the $90 \%$ confidence interval around the correlation between identification and amotivation did not encompass unity. Furthermore, we conducted a comparison between the 5-factor model and a more constrained nested model with the correlation between identification and amotivation fixed to unity, thereby simulating a 4-factor model with identification and amotivation items as indicators of a single latent variable. The results of a $\chi^{2}$ difference test showed that the 4-factor model fitted significantly worse than the 5-factor model $\left(\chi^{2}\right.$ difference $\left.=56.23, \mathrm{df}=1\right)$. Thus the evidence suggests that the identification and amotivation subscales are assessing different constructs.

Wilson et al. (2002) tested a true simplex model of the BREQ as well as a correlated factor model by specifying causal paths among the constructs. We chose not to adopt this approach because such a model implies a causal chain among the behavioral regulations with amotivation causing external regulation, which in turn causes introjection, and so on. We do not believe such a model is theoretically justified. Rather, the theory carries the less stringent assumption that the constructs are intercorrelated in an ordered pattern as described above. 
In conclusion, this study has provided support for the factorial validity of the BREQ-2. While some caution and further research is warranted given the distributional properties of the amotivation items, and indeed the external regulation items, the BREQ-2 could allow for a more complete assessment of motivation from the SDT perspective and for the investigation of the antecedents and consequences of amotivation for exercise.

\section{References}

Deci, E.L., \& Ryan, R.M. (1985). Intrinsic motivation and self-determination in human behavior. New York: Plenum.

Deci, E.L., \& Ryan, R.M. (1991). A motivational approach to self: Integration in personality. In R. Dienstbier (Ed.), Nebraska symposium on motivation. Vol. 38: Perspectives on motivation (pp. 237-288). Lincoln: University of Nebraska Press.

Godin, G., \& Shephard, R.J. (1985). A simple method to assess exercise behavior in the community. Canadian Journal of Applied Sport Sciences, 10, 141-146.

Mullan, E., \& Markland, D. (1997). Variations in self-determination across the stages of change for exercise in adults. Motivation and Emotion, 21, 349-362.

Mullan, E., Markland, D., \& Ingledew, D.K. (1997). A graded conceptualisation of selfdetermination in the regulation of exercise behaviour: Development of a measure using confirmatory factor analytic procedures. Personality and Individual Differences, 23, 745-752.

Rose, E.A., Markland, D., \& Parfitt, G. (2001). The development and initial validation of the Exercise Causality Orientations Scale. Journal of Sports Sciences, 19, 445-462.

Ryan, R.M., \& Connell, J.P. (1989) Perceived locus of causality and internalization: Examining reasons for acting in two domains. Journal of Personality and Social Psychology, 57, 749-761.

Ryan, R.M., \& Deci, E.L. (2000). Self-determination theory and the facilitation of intrinsic motivation, social development, and well-being. American Psychologist, 55, 68-78.

Satorra, A., \& Bentler, P.M (1994). Corrections to test statistic and standard errors in covariance structure analysis. In A. Van Eye \& C.C. Clogg (Eds.), Analysis of latent variables in developmental research (pp. 399-419). Newbury Park, CA: Sage.

Wilson, P.M., Rodgers, W.M., \& Fraser, S.N. (2002). Examining the psychometric properties of the Behavioral Regulation in Exercise Questionnaire. Measurement in Physical Education and Exercise Science 6, 1-21.

Wilson, P.M., Rodgers, W.M., Blanchard, C., \& Gesell, J. (2003). The relationship between psychological needs, self-determined motivation, exercise attitudes, and physical fitness. Journal of Applied Social Psychology, 11, 2373-2392.

\section{Author Note}

The BREQ-2 can be downloaded from:

http://www.bangor.ac.uk/ pes004/exercise_motivation/scales.htm

Manuscript submitted: February 17, 2003

Revision accepted: July 15, 2003 